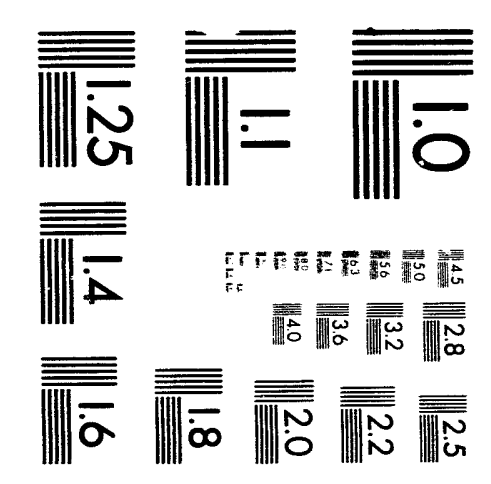



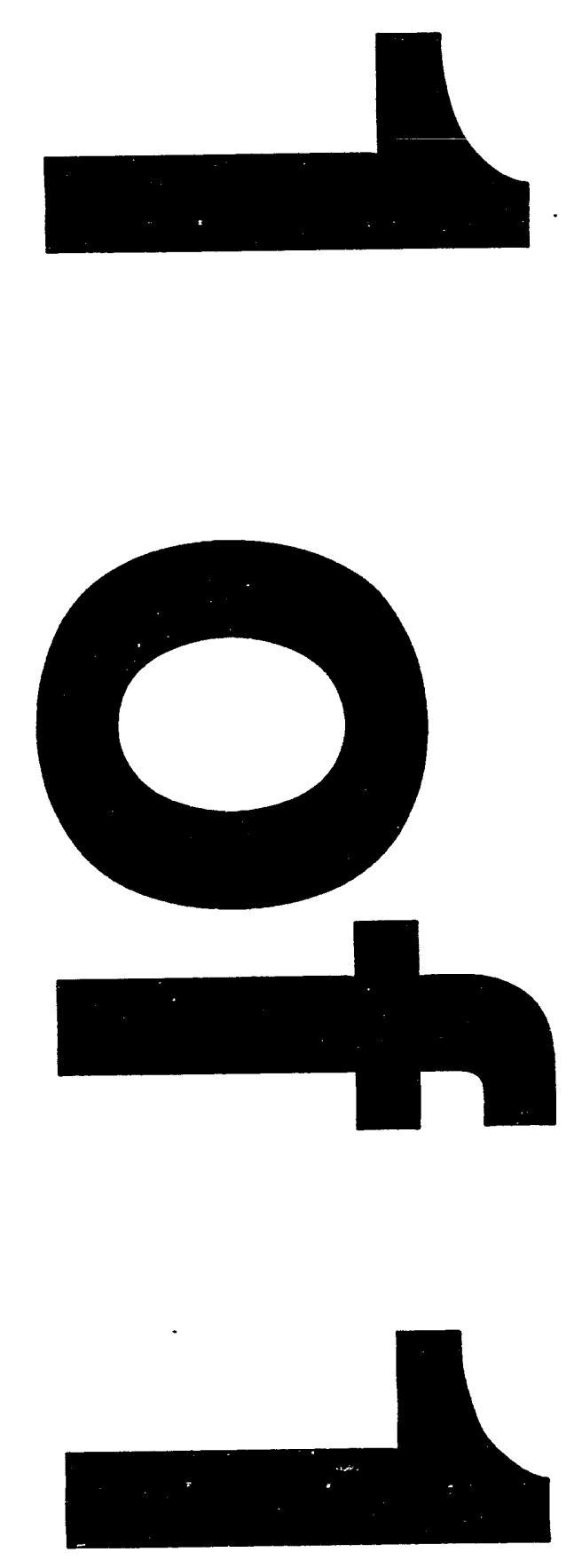
SAND92-2909

Distribution

Unlimited Release

Category UC-706

Printed October 1993

\title{
Holographic Interferometry:
}

\section{A User's Guide}

\author{
Donovan Griggs \\ Electrical Standards Department \\ Sandia National Laboratories \\ Albuquerque, NM 87185
}

\begin{abstract}
This manual describes the procedures and components necessary to produce a holographic interferogram of a flow field in the Sandia National Laboratories hypersonic wind tunnel. In contrast to classical interferometry, holographic interferometry records the amplitude and phase distribution of a lightwave passing through the flow field at some instant of time. This info:mation can then be reconstructed outside the wind tunnel for visual analysis and digital processing, yielding precise characterizations of aerodynamic phenomena. The reconstruction and subsequent hologram image storage process is discussed, with particular attention paid to the digital image processor and the data reduction technique.
\end{abstract}

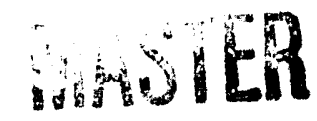




\section{Contents}

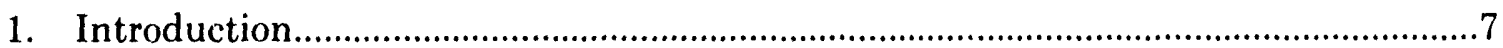

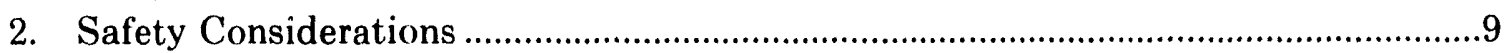

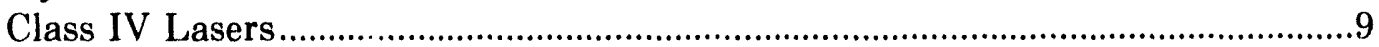

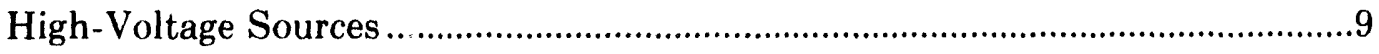

Photographic Processing Chemicals.....................................................................10

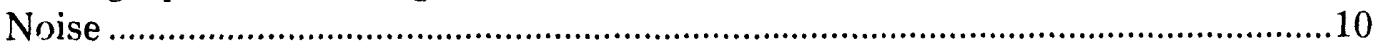

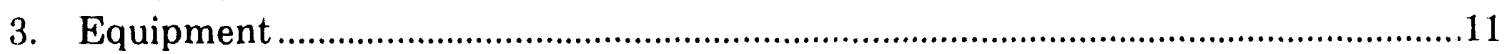

Nd:YAG Fulsed Laser ..................................................................................11

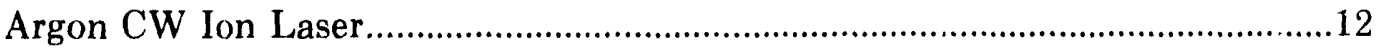

Photographic Glass Plate.....................................................................................12

Developer and Associated Photoprocessing Chemicals ........................................12

Construction and Reconstruction Optics ............................................................13

CCD Camera and Photographing Station ...........................................................13

Image Processing System.......................................................................................13

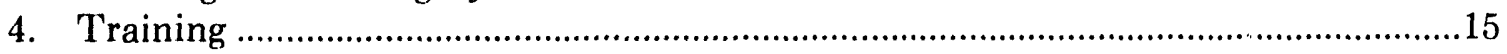

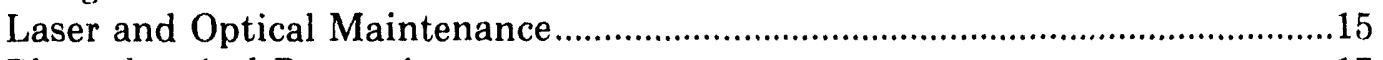

Photochemical Processing.......................................................................................17

Image Processing ...............................................................................................18

5. Holographic Interferometry Procedures ..............................................................19

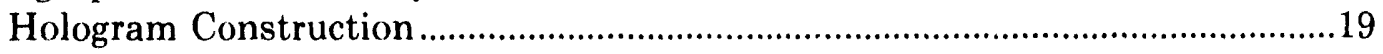

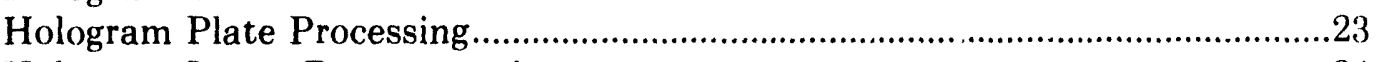

Hologram Image Reconstruction ........................................................................22

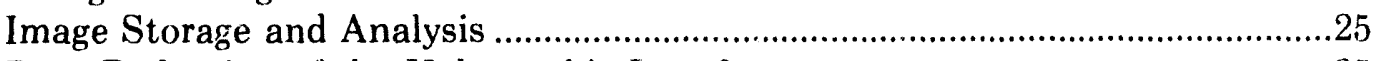

Data Reduction of the Holographic Interferogram.............................................25

6. Problem Areas in Holographic Interferometry …………………………………......27

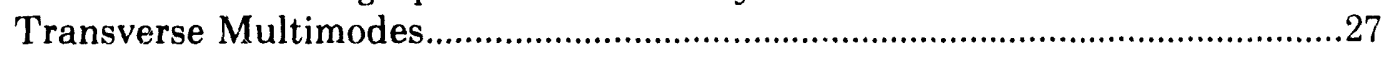

Laser Rod and Component Temperature …………………………………......2

Relative Ligh ¿ness and Darkness .....................................................................2

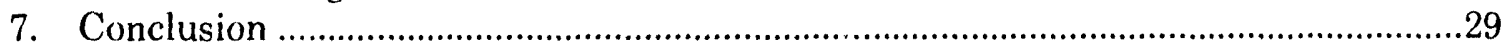

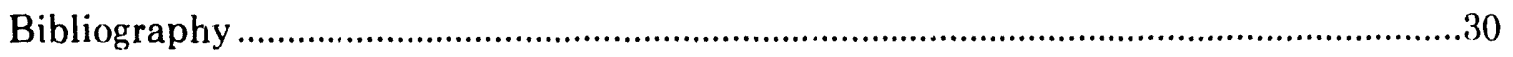

\section{Figures}

1. Projection of an object field with an opaque object.................................................

2 Reference beam optics shown in top view …………….........................................20

3 Object beam optics shown in view along wind tunnel ............................................20

4 Hologram recording system..................................................................................21

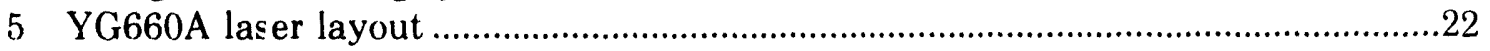

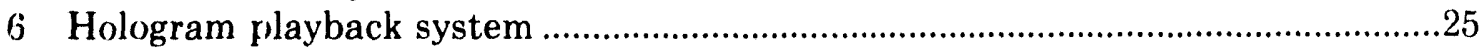

7 Hologram image and fringe pattern....................................................................26

8 Relationship of physical and Fourier planes ..........................................................26

9 Transverse modes in a laser cavity .......................................................................28 


\section{Holographic Interferometry: A User's Guide}

\section{Introduction}

This manual describes the procedures and components necessary to produce a holographic interferogram of a flow field in the Sandia National Laboratories (SNL) 18 in. hypersonic wind tunnel. Reference to a commercial product does not imply endorsement by Sandia National Laboratories or the Department of Energy or the lack of a suitable substitute.

Classical interferometry uses the mixing of two coherent waves to measure the distortion in one of the waves. Usually this information is used to measure optical path changes, either by transmission through or by reflection from an optical quality element. Consequently, optical components used in assembling a classical interferometer must be fabricated with a high degree of precision so that they do not contribute spurious fringe patterns.

In contrast to classical interferometry (Caulfield, 1979), holographic interferometry also records the amplitude and phase distribution of a lightwave passing through the flow field at some instant of time. This information can then be reconstructed outside the wind tunnel for visual analysis and digital processing, yielding precise characterizations of aerodynamic phenomena.

The holographic interferometer described in this manual is a variation of a wave-front-dividing interferometer such as the Mach-Zehnder and Michelson interferometer (Caulfield, 1979). This system uses beamsplitters to separate a plane wave into two paths: One passes through the test region, generating an object field; the other is unobstructed and acts as a reference field (Figure 1). Instead of using a second beamsplitter to recombine the two beams, a hologram is used. At this point a holographic image is produced and may be photographed if desired. Alternatively, a dual-plate, double-exposure method may be employed. One exposure is taken with a uniform density field, and a specified time later another exposure is taken with a nonuniform density field. When the disturbed and undisturbed object waves are simultaneously reconstructed, they interfere to form the desired fringe pattern. The dual-plate, double-exposure method is described in this manual. 
In conjunction with the interferometric hologram construction technique described above, the reconstruction and subsequent hologram image storage process is discussed. Particular attention is paid to the digital image processor and the data reduction technique.

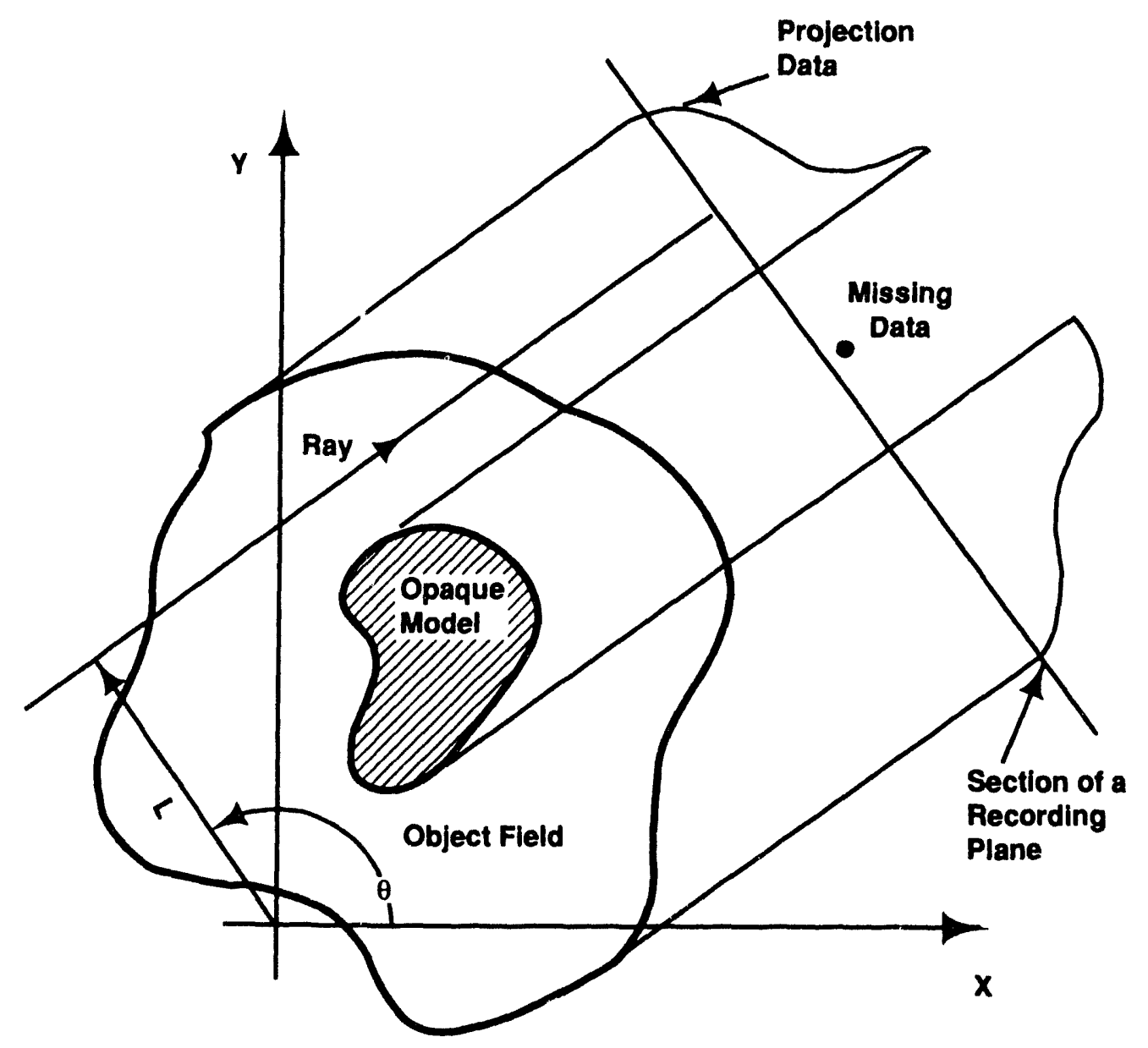

Figure 1. Projection of an object field with an opaque object. 


\section{Safety Considerations}

The holographic interferometry technique discussed here involves a number of specialized systems that expose personnel to a considerable amount of hazard and therefore dictate a need to discuss specific training and safety considerations.

Operation of the entire system is covered in ES\&H SOP 11800-8904 (Doerr, no date). Safety concerns in the following four areas are summarized in this section.

1. Class IV lasers used for hologram construction and reconstruction

2. High-voltage sources used for the laser systems

3. Photographic processing chemicals

4. Noise

\section{Class IV Lasers}

First and most important are the Class IV lasers that are used for hologram construction and the subsequent illumination for image analysis and storage These systems are capable of producing laser radiation that can cause severe burns and permanent eye damage from a direct or diffusely reflected laser beam entering the eye. Also, direct exposure of the beam to the skin can cause uncomfortable burns. For these reasons personnel working with lasers must attend the workplace hazards training course in laser safety, conducted by the Industrial Hygiene Department (7711). Also, before starting any lasing, Sandia requires that all personnel take a retinal examination by a qualified ophthalmologist.

All laser safe operating procedures, general and specific to the holographic interferometry technique, should be strictly followed at all times. These procedures include inspecting the laser proiective eyeware by the primary laser operator before initial laser operation to ensure that the eyeware will sufficiently reject the entire bandwidth of laser radiation emitted from the laser unit. Anytime the laser is to be energized, protective eyeware is to be worn. It is essential never to look directly into the path of a laser beam, even with protective glasses.

\section{High-Voltage Sources}

The second area of corcern is the existence of high-voltage sources in and around laser components. These voltages range from 300 to $800 \mathrm{Vdc}, 220$ to $600 \mathrm{Vac}$, with a potential for capacitor discharge from 6 to $10 \mathrm{kV}$. All potentials may be lethal; therefore, strict training and compliance with general high-voltage electrical safety and all procedural guidelines are mandatory. Maintenance on any components should be carried out by qualified personnel only. 


\section{Photographic Processing Chemicals}

The third area of concern is the potential hazard that exists in developing the photographic plates used as the hologram recording material. The chemicals used are corrosive and can cause burns on the skin and possible vision damage if splattered into the eyes. All of the photoprocessing is done in total or partial darkness, which increases the potential for spills. Therefore, hands-on training and familiarity with the developing process is essential.

\section{Noise}

Finally, the holographic interferometry process may dictate that personnel are very near the wind tunnel during operation. This activity exposes personnel to a very high noise level. Although noise levels are not hazardous over short exposure times, they can be uncomfortable and can change suddenly. Therefore, ear protection is strongly recommended. 


\section{Equipment}

This section describes the systems used to perform holographic interferometry in Sandia's 18 in. hypersonic wind tunnel. Variations in equipment specifications can be made and will necessarily depend on actual test parameters. For more detailed information pertaining to subassemblies, see the Model YG660A Laser Technical Manual (Quantel International, 1988), referred to in the bibliography at the end of this manual.

These systems include the following:

- Nd:YAG pulsed laser

- Argon CW ion laser

- 8E56 silver halide emulsion photographic glass plate

- D-19 developer and associated photoprocessing chemicals

- Construction and reconstruction optics

- CCD camera and photographing station

- VisionLab A'T-based image processor and Imagepro II system software

\section{Nd:YAG Pulsed Laser}

The laser system recommended for hologram construction is an actively $Q$-switched Nd:YAG Pulse Laser Model YG660A. Pulse repetition rate is $10 \mathrm{~Hz}$, with a pulse width of $10 \mathrm{~ns}$. With a second harmonic generator, the nominal beam energy is $145 \mathrm{~mJ}$ at a wavelength of $532 \mathrm{~nm}$ (half of the normal frequency for a Nd:YAG laser). A linewidth narrowing Etalon (LNE) is attached to minimize linewidth. The YG660A has four modes of operation:

1. External trigger

2. Manual trigger

3. Fixed repetition rate

4. Variable repetition rate

The total system, including support systems, consists of the following:

- Laser head

- Power units, PU610 and PU620

- Simmer capacitor banks, CB630

- Supply box, SB660

- Logic unit, LU660

- Cooling group, CG603

- Q-switch, Qs410

The lasing device used in the laser head is a YAG (yttrium aluminum garnet) crystal $6 \mathrm{~mm}$ in diameter, optically pumped by one flashlamp in an enclosed, coupled configuration. The Q-switch consists of a Pockels cell, quarter wave plate, dielectric polarizer prism, and Q-switch electronics. The Model YG660A requires a $208 \mathrm{Vac}, 30 \mathrm{~A}$, three phase power source. A tap water outlet with a volume of 1 to $2 \mathrm{gal} / \mathrm{min}$ is needed at 30 to 50 psi maximum for heat exchanger CG603. 


\section{Argon CW lon Laser}

For hologram illumination, a Model 164 argon ion laser with a Model 265 exciter is recommended. 'The continuous output power is $5 \mathrm{~W}$, which is distributed over 514.5 to $454.5 \mathrm{~nm}$. The heart of the Model 164 ion laser is a BeO-bore plasma tube. The Model 164 requires a $208 \mathrm{Vac}$. $30 \mathrm{~A}$, three phase power source. Filtered tap water, at $2 \mathrm{gal} / \mathrm{min}$, is required for cooling.

\section{Photographic Glass Plate}

The suggested hologram material is a silver halide emulsion glass plate with an emulsion thickness of $7 \mu \mathrm{m}$. 'This material has a light sensitivity of 400 to $550 \mathrm{~nm}$, ideal for use with the $532 \mathrm{~nm}$ wavelength from the Nd:YAG laser. An 8E56 plate usually comes in a $4 \times 5 \mathrm{in}$. area, but larger areas are available. The $8 \mathrm{E} 56$ requires $30 \mu \mathrm{J} / \mathrm{cm}^{2}$ for exposure. This is about midrange for light intensity requirements.

\section{Developer and Associated Photoprocessing Chemicals}

The processing chemicals necessary to develop an 8E56 type photographic plate are as follows (Smith, 1977):

- Kodak developer D-19 (single powder)

- Kodak indicator stop bath

- Kodak rapid fixer, parts A and B

- Kodak Photo-Flo 200 solution

D-19. The principal components of D-19 are sodium sulfite, sodium carbonate, hydroquinone, and p-methylaminophenol sulfate. This mixture is a powder and comes in a packet. When mixed with water, it makes a gallon of developer. Because D-19 is temperature and light sensitive, it should be stored at room temperature in an opaque container. In spite of these precautions, the shelf life of the mixed product is not more than a month. Use it within that time or discard it.

Stop Bath. The indicator stop bath is a dilute solution of acetic acid, which should be yellow. If it is not, discard it. Usually it is very stable and has an indefinite shelf life. It is used to stop the developing process within the emulsion.

Rapid Fixer. After you stop the developing reaction, you must fix the silver atoms (the darkened areas) in the emulsion by means of the rapid fixer. This is a two part mixture (part A and part B). Part A consists of the following:

- Water

- Ammonium thiosulfate

- Sodium acetate

- Boric acid

Rapid fixer part $B$ consists of the following:

- Water

- Aluminum sulfate

- Sulfuric acid

Photo-Flo 200. To ensure a streak-free plate, use Photo-Flo 200. This product consists of water, propylene glycol, and p-tertiary-octylphenoxy polyethyl alcohol. 
Material safety data sheets for all chemicals are in all appropriate departmental MSISS file's.

\section{Construction and Reconstruction Optics}

As mentioned in the Introduction, the holographic interferometry procedure discussed here involves hologram construction and reconstruction processes. The optics required for both processes are similar, must all be chosen to transmit or reflect in the 400 to $700 \mathrm{~nm}$ bandwidth, and should be of high laser power quality. 'They consist of the following:

- High power laser mirrors $10(2 \mathrm{M} 20$ and $20(2 \mathrm{M} 20$

- Broadhand infrared reflector 6(0) 20 and 601)10 with ER.2 coating

- All band high reflector $\mathrm{BI}) .1$

- Bi-concave optical glass lens 01LDKO(0)5

- Plano-concave optical glass lenses 011.PK(0)1, 011,PK007, KP(025, KPC022

- Oriel Model 42740 achromatic collimating lenses

- Spatial filters

- Film plate holders Models 5:35 and 520

- Model AB5505i3H2 beamsplitter

\section{CCD Camera and Photographing Station}

For image reconstruction, the following two items are necessary:

- PZ-150F Burleigh driver and associated micropositioners

- Cohu Model 4865-70(0) C(DI) video camera

\section{Image Processing System}

The Visionlab II advanced technology (A'T)-based system was used for digital image processing. This system consists of the following:

- IBM PC: A'T'-compatible A'T' card

- 3M Comtal frame processor (FPI)

- 3M Comtal display processor (I)PI)

- ImagePro II image processing software

AT Card. 'The A'T card is 100"; IBM compatible and contains an 80286 16-bit microprocessor that runs at 8 or $12 \mathrm{MHz}$. An 80287 coprocessor is optional. The card has a clock and calendar with battery backup and contains 1 megabyte (MB) of random access memory (RAM).

Frame Processor. The $3 \mathrm{M}$ Comtal frame processor provides real-time image capture from a monochrome camera, broadcast video [American National Television Standard Committee (N'TSC) format or British (PAL) format), VCR, slow scan device, or digital output device. 'This processor allows capture of a full $512 \times 512$ color image in $0.1 \mathrm{s.}$

Display Processor. Visionl ab II uses a digital display processor for image storage and display. 'This board has small, round, gold connectors located on the end plate. Visionlab II is mapped into IBM memory segment hexidecimal $\mathrm{D}(0) 0 \mathrm{H}$; the input/ output $(1 / \mathrm{O})$ address is $0300 \mathrm{H}$. 'The image memory is mapped as $64-\mathrm{kB}$ blocks into the $\mathrm{D} 000 \mathrm{H}$ segment window. 
Image Processing Software. ImagePro II image processing system by Media Cybernetics was the software used for image manipulation. With this system, hologram images may be captured, stored, or retrieved from mass storage. While an image is in video memory (displayed on the monitor), a number of operations may be performed:

- Contrast adjustments

- Filtering

- Composite image subtraction and addition

- Area and line histograms

- Line profile

- Gray-scale color assignments

- Line fast Fourier transform (FFT)

A full $512 \times 512$ field Fourier transform option is also available that will allow construction of tailor-made digital filters. These functions are easily accessible through pull-down menus and a mouse positioner. The software package is completely written in C programming language. 


\section{Training}

Holographic interferometry as an analytical technique for aerodynamic processes in wind tumnels has been under development for some time (Caulfield, 1979); thus, the technology is not new in the field of holography. Intil the advent of digital image processing systems, however, the actual practice of holographic interferometry for the purpose of flow field analysis has been minimal. As a result, some training or pretest familiarity is necessiry in three major areas: laser and optical maintenance, photochemical processing, and image processing.

\section{Laser and Optical Maintenance}

As mentioned earlier, holographic interferometry requires less stringent optical and laser system specifications than classical interferometry, but some laser beam specifications should be considered.

\section{Laser Beam Requirements}

First, the radial beam intensity distribution should be a Caussian distribution because the Fourier transform of this distribution pattern is also Caussian. 'Thus, the distribution remains Gaussian at every point along its path of propagation through the optical system.

Most lasers automatically oscillate with a Caussian distribution, but since spatial filtering may be required to achieve a circular beam, care must be taken in cutting off the (iaussian distribution with a very small aperture. This will cause non-Ciaussian beam features. An aperture diameter of 3 to 4 times the (iaussian beam radius is sufficient. 'These beam requirements are necessary for hologram construction as well as reconstruction.

The laser performance is determined by specified parameters such as Ciaussian beam distribution, beam geometry, and beam energy. Beam energy is of considerable importance and involves the area that demands the most attention from laser operators. 'This parameter is important since the photographic emulsion has a minimum energy limit for proper exposure. To achieve maximum beam energy, the laser head and resonance cavity must be properly aligned. 'The spatial beam distribution (beam geometry) is important for holography and also comprises a measure of laser performance.

The performance energy value can be obtained by using a suitable meter (such as Scientech Model 362). 'To quickly monitor the spatial distribution, make burn patterns by using Kodak Linagraph sensitive paper. If either the energy or spatial distribution parameter are unsatisfactory, switch the laser off and remove the main cover. Check the $1064 \mathrm{~nm}$ energy and spatial distribution between the second harmonic generator and the quarter wave plate. If the beam is not round or the energy is low, align the laser. A description of the alignment procedure is given in the next section.

Another condition that will cause a gradual loss of beam energy is dirty laser cavity optics. 'The laser cavity consists of a number of turning mirrors and quarter wave plates. 


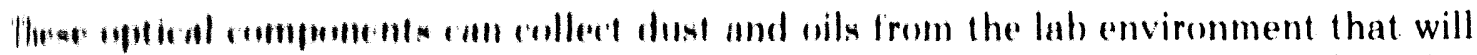

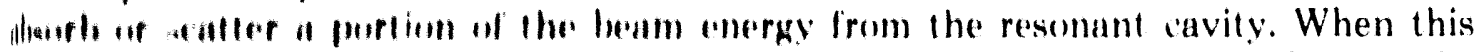

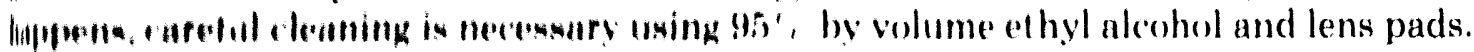

\section{8wlichod Pookele Coll}

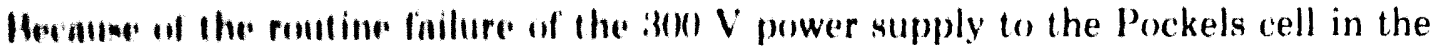

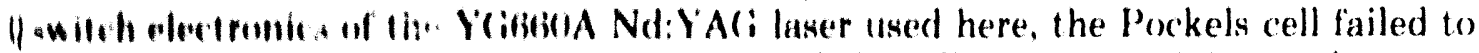

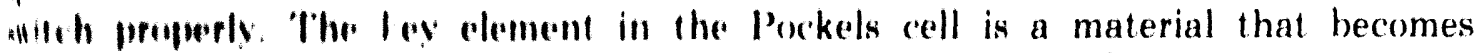
lim frinuent under lhe influence of un extermal electric field. 'The birefringent crystal is

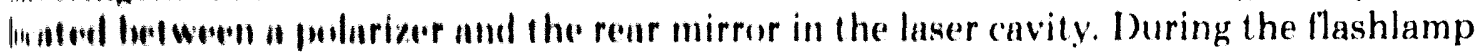

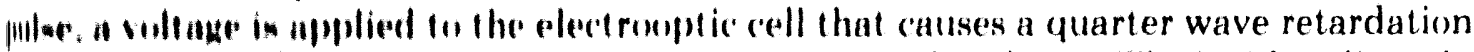
fincurn the and y milurizulion components of the inciden: beam. The incident linearly fularionl linhe is circularly pularized after passing through the Q-swich crystal. After lumg follon lend Irum the mirror, the radiation again passes through the electrooptic cell

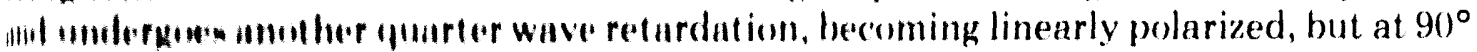

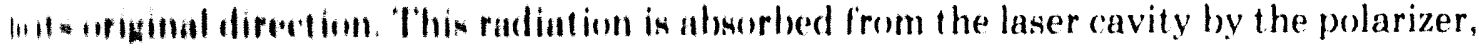

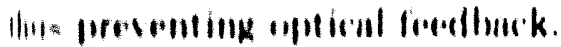

linuril the and in the flimhlump) pulse, the voltage on the cell is electronically

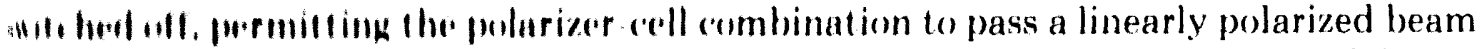
"Hillunt lome lecillulion within the cavity will build up, and after a short deiay, a

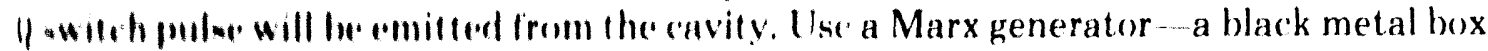

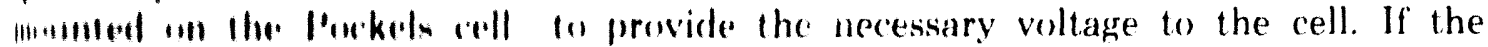
Hewrolur lailo lo prouluse this volluge, making lasing impossible, replace the Marx Hintenthir

\section{Coollng Wator Filtor}

Amolher compunnent hint reguires periodic atlention is the deionized water filter.

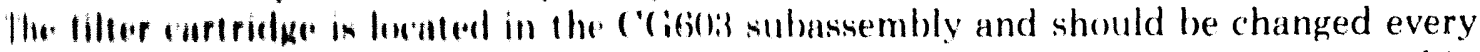

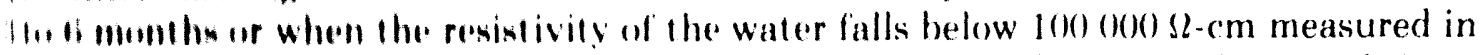

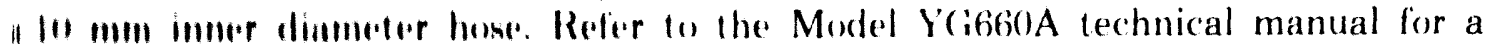
intailend prociediers.

\section{LWo80 8ecurliy Systom}

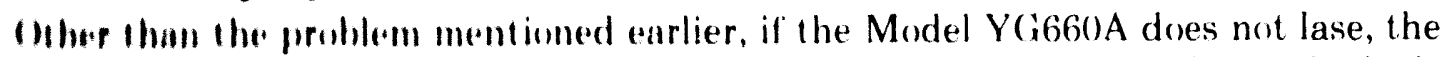

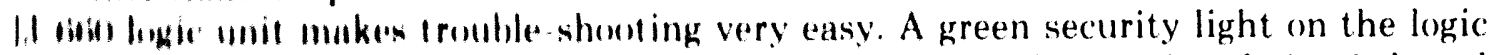
Imm imliculen a prohlem in the system. Refer to the 1,0660 logic printed circuit board

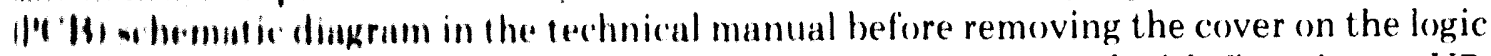

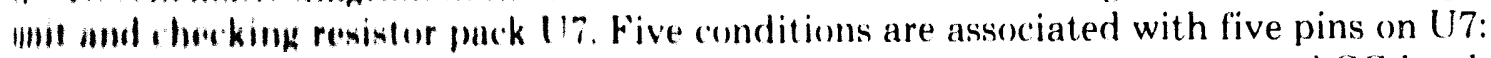

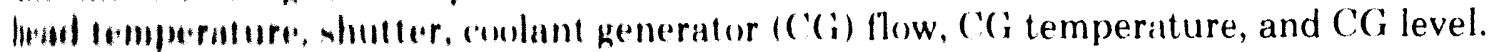
II ails ill Ihes. comblitions are oul of the normal operating specification, the green light

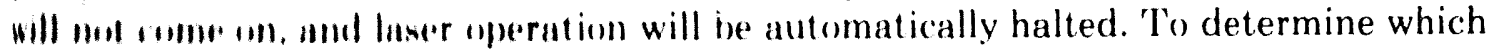
Whe liw. in comsing lhe problem, use a voltmeter to measure the voltage on the five pins IIII: II th Vile in mol prosent, this system has a fault.

\section{Caullon: Aemove any metal objects from hands and forearms to reduce} the pousbility of electrical shock. 


\section{Argon Ion Laser}

The Model 164 argon ion laser requires little maintenance, but if it has not been operated for a long time, the plasma tube may need to be aligned. Alignment is accomplished by bringing the optics and the plasma tube to a line determined by the resonator structure and the aperture. Alignment requires four adjustments:

- Vertical positioning at the cathode end of the tube

- Vertical positioning at the anode end of the tube

- Horizontal positioning at the cathode end of the tube

- Horizontal positioning at the anode end of the tube.

Make all of these adjustments while monitoring the laser output power. Adjust each for maximum output.

Similar to the Model YG660A, the Model 164 with an exciter has a water cooling system that requires periodic replacement of a water line filter. If the water circulating through the plasma tube looks milky or shows particulates, replace the filter, which is located in the bottom of the 265 exciter.

\section{Optics}

Clean the optics required to direct the reference and object beams to the hologram only when it is absolutely necessary because accidental contamination of mirror or lens surfaces can easily happen when cleaning occurs. Dusting with dry air is preferable but, if necessary, $95^{\circ} \circ$ by volume ethyl alcohol may be applied with lens pads. To limit the amount of dust and contamination, cover or store all optical components in plastic bags or containers.

\section{Photochemical Processing}

Photochemical processing is certainly not a new technology, but as mentioned, the specific requirements of working with hazardous chemicals in limited or total darkness necessitate training and familiarity with this area.

A detailed description of the mixing of photochemicals is given in Section 5, Holographic Interferometry Procedures. It is important that the D-19 powder be mixed with water at a temperature warmer than room temperature. Allow it to cool to room temperature $\left(21.6\right.$ to $\left.25^{\circ} \mathrm{C}\right)$ to consistently reproduce developments of the $8 \mathrm{E} 56$ silver halide emulsions. If the solution is used at elevated temperatures, development will proceed faster, and the resultant hologram will be much darker. This will affect the transmittance of the hologram and will make the final image production, during reconstruction, very difficult.

With room temperature D-19 solutions, it is important to correlate the length of time the plate is exposed to the construction heam with the length of time the plate is immersed in the developing solution. This correlation may be difficult to establish because the specific flow field conditions change the amount of light that reaches the plate during construction. Therefore, trial and error may be needed before acquiring the specific correlation. 


\section{Image Processing}

The image processing system and software used for the holographic interferometry procedure, discussed later, is an AT-based microcomputer that accesses and manipulates the Comtal frame processor (FPI) and display processor cards inside the system chassis. These cards may be accessed by the following five methods:

- Imagepro image processing software

- Comtal executive control drivers and utilities

- Stand-alone programs that make calls to Comtal executive control drivers

- Stand-alone C-language programs that call Imagepro functions

- C-language routines incorporated into Imagepro users modules.

Generally, only Imagepro software is needed for holographic interferogram analysis and fringe interpretation, but if more analytical techniques are needed, Fortran IV, IBM Basic, Pascal, or C languages can be used. The Imagepro software system has the advantage of using mouse-operated, pull-down menus, which make the software easy to manipulate. 


\section{Holographic Interferometry Procedures}

The holographic interferometry performed at the SNL hypersonic wind tunnel includes five activities:

- Hologram construction

- Hologram plate processing

- Hologram image reconstruction

- Image storage and analysis

- Data reduction.

Some of these activities involve environment, safety, and health (ES\&H) considerations and require specific safe operating procedures (SOPs). Therefore, required SOPs are listed in the paragraphs that follow.

\section{Hologram Construction}

\section{Optical Setup for Object and Reference Beam Projections}

First, hologram construction requires an optical setup for object beam and reference beam projections. Figures 2, 3, and 4 show the proper optical arrangement for these projections. In Figure 2, L1 is a plano-concave lens, and L 2 is a plano-convex lens. 'They are arranged to collimate the emerging beam so that the spatial beam configuration can be maintained over longer distances. The proper focal length must be chosen for each lens. Figure 3 shows lenses $L 3$ and L4 in the object beam path. These lenses are used for the final beam collimation before passing through the wind tunnel test section. The diameter and focal length of L4 will determine the hologram plate size and the field of measurement in the test section. Figures 2 and 3 also show the side and top view of the reference path with lenses $L 5$ and $L 6$, which are the final collimating optics for the reference beam.

Use mirrors M1 to M10, which are high-power, first-surface mirrors, to direct the collimated beam to the test section and the hologram plate. M3 and M4 are part of the beam-steering mirror assembly Model 765. Use the large, 6-in.-dia mirrors M5 and M6, Models 60D20 and 6()D10, in the object beam to direct the 3-in.-dia collimated beam through the test section and onto the hologram. Note the zig-zag pattern in the reference path in Figure 3. This pattern is necessary to allow object and reference path lengths to be equal when interfering on the hologram. In this way, any temporal differences in object and reference beam wave fronts are due to wind tunnel flow field density variations. The area outside the wind tunnel may dictate that the configuration of these paths differ greatly. It is important to measure these paths accurately. Use a measuring device of known length for measurements. Since the wind tunnel test section will absorb and deflect a portion of the object beam, adjust the beamsplitter (BS), Model $\mathrm{AB} 35.5053 \mathrm{H} 2$, in such a way that the reference beam intensity is one half the object beam intensity. 


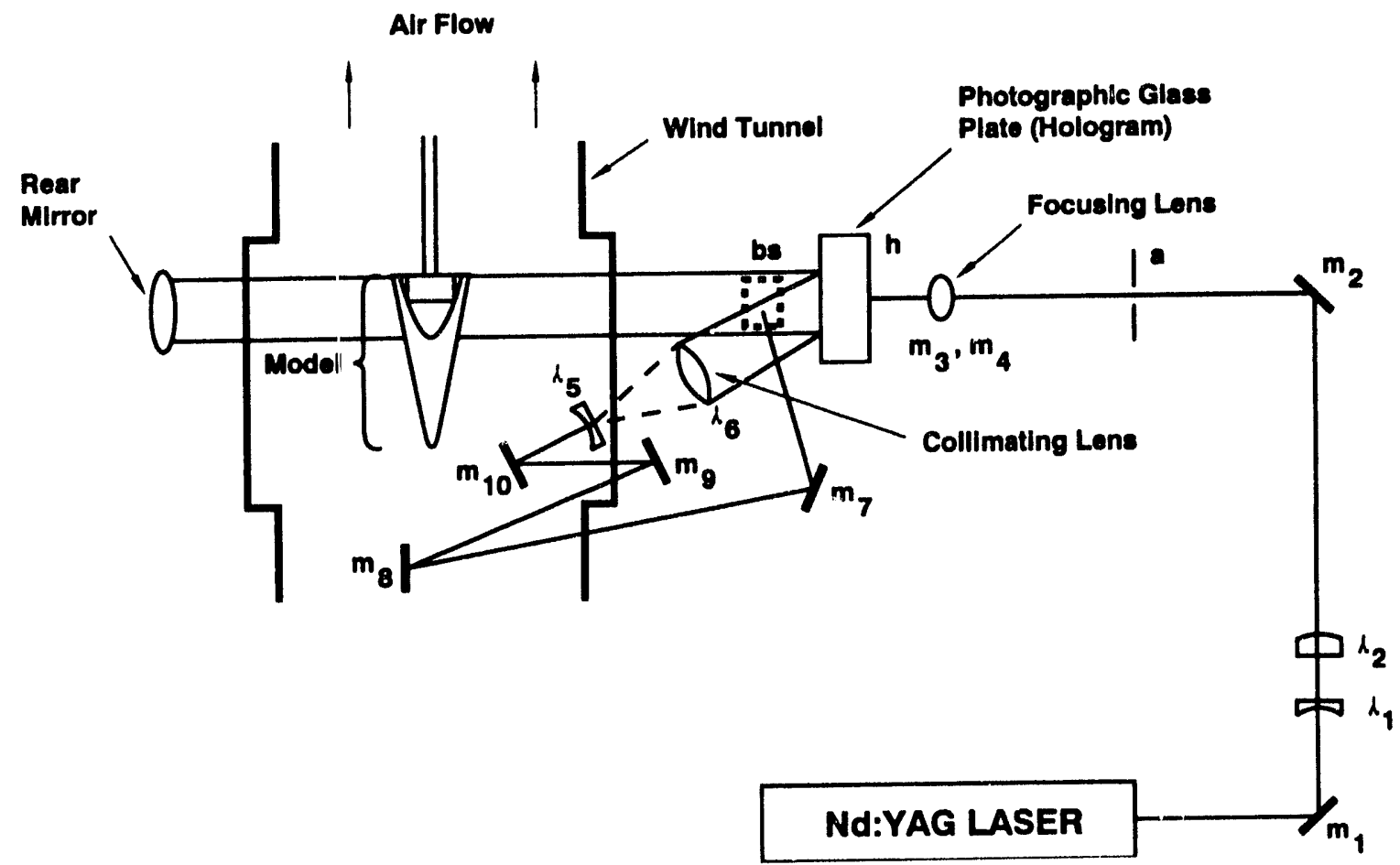

Figure 2. Reference beam optics shown in top view.

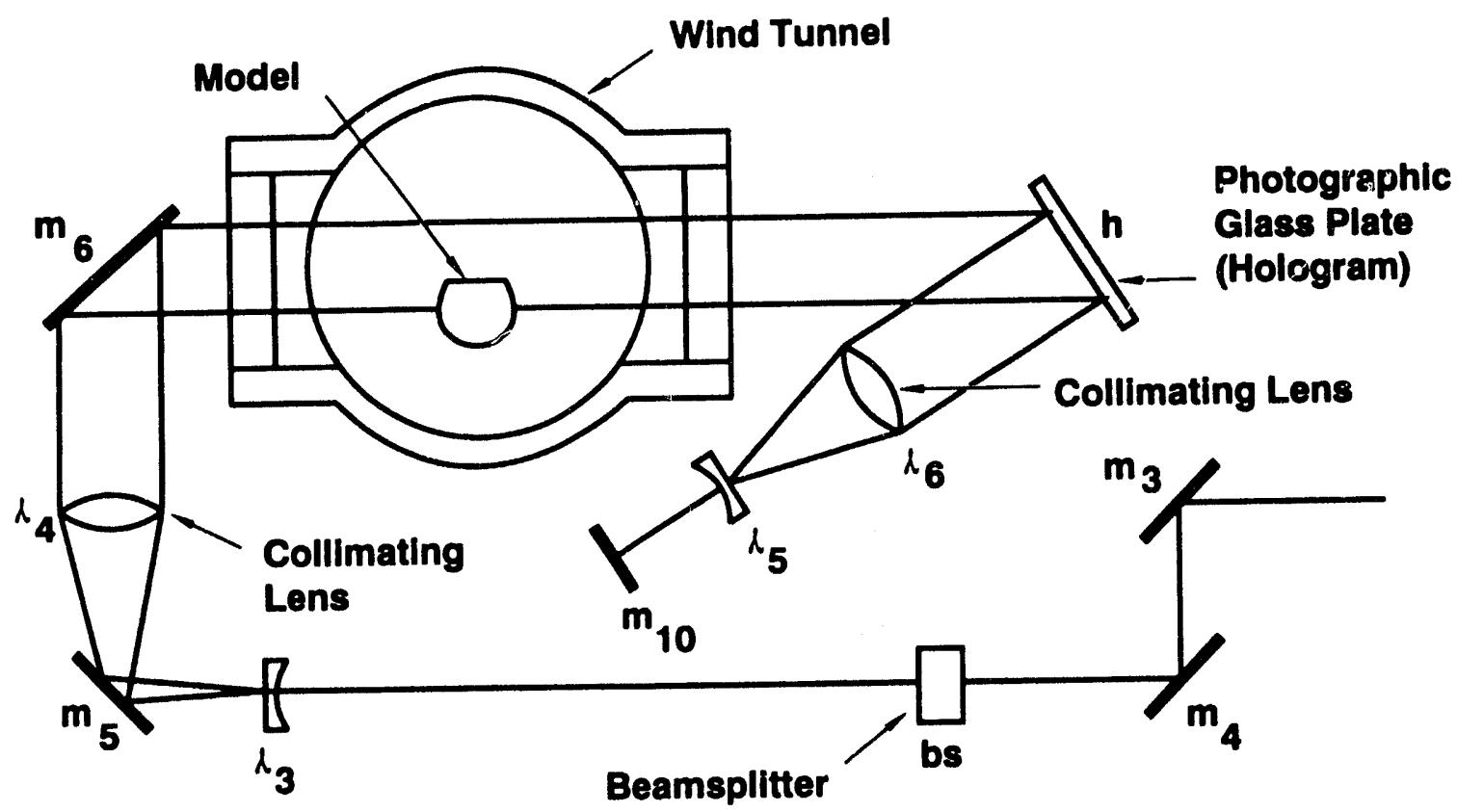

Figure 3. Object beam optics shown in view along wind tunnel. 


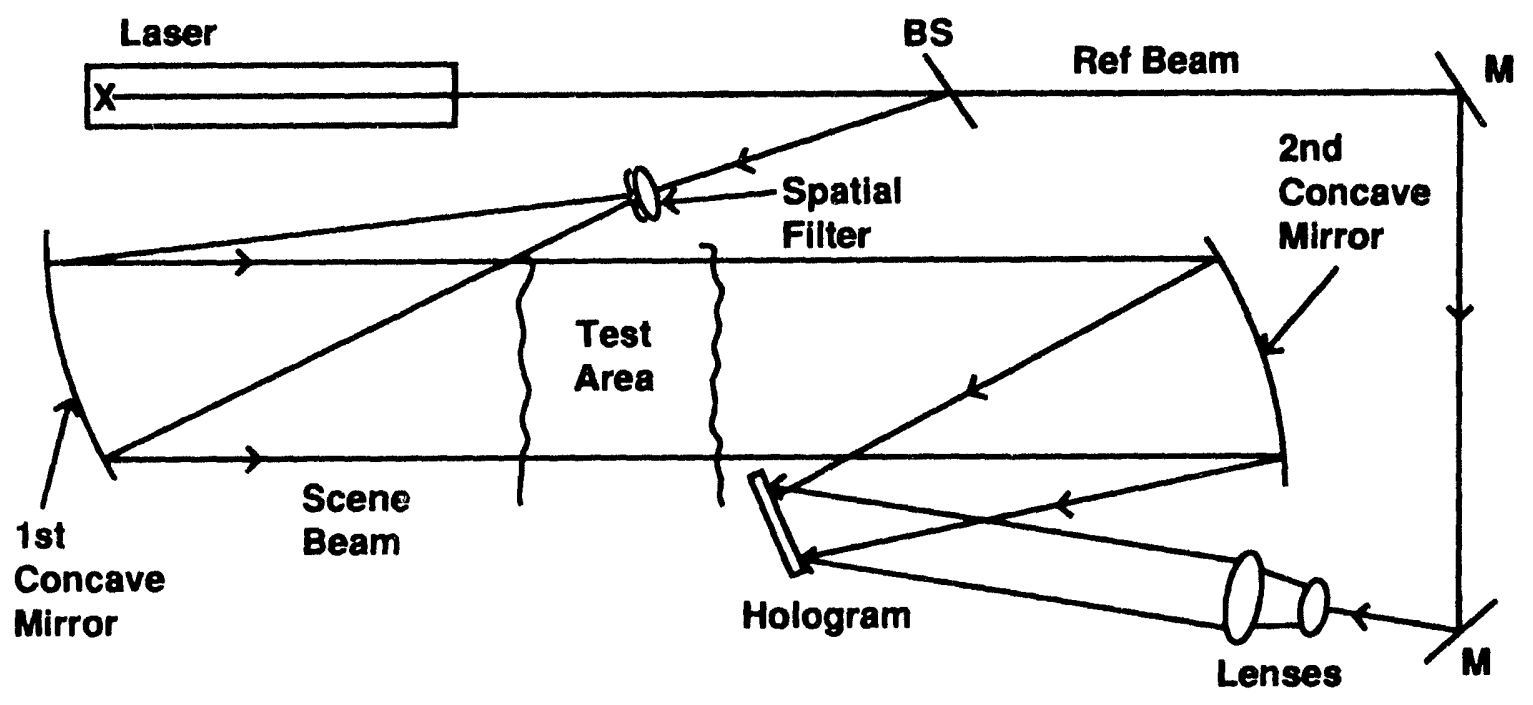

Figure 4. Hologram recording system.

A spatial filter (pin hole "a" in Figure 2), used to further improve beam geometry, is optional. Its use is determined by the initial beam quality emitted from the laser.

After completing the above setup, use the YG660A Nd:YAG laser to perform the final optical component alignment; alternatively, a low-power $\mathrm{HeNe}$ laser may be used. This procedure requires a Class IV laser SOP and construction of a laser enclosure to protect personnel without protective eyeware from stray laser radiation. The door to the enclosure must be interlocked with the YG660A Nd:YAG laser in such a way that the laser is inoperable when the door is open. The enclosure should cover $360^{\circ}$ around the laser area to ensure that no stray beam can enter the outer lab area. SOP No. 118008904 is the proper Class IV laser SOP that must be read and signed before op rating the laser.

With the laser on, verify proper alignment of the optical components by placing a piece of paper in the hologram holder. With the reference beam blocked, check for a homogeneous, illuminated circular spot at the center of the paper. A shadow of the model in the wind tunnel test section should be uniformly positioned in this spot on the paper. Now, block the object beam and allow the reference beam to illuminate the paper. Once again, a uniform, illuminated circular spot should be seen on the paper but without the test section shadows produced from the test model. Finally, allow both beams to pass to the paper and align each beam to coincide in the middle of the paper.

\section{Nd:YAG Laser Alignment}

After the laser has been aligned for peak energy output, an exposure can be taken. The procedure for aligning the Model YG660A Nd:YAG laser is as follows:

1. If the beam geometry is circular but has low energy output, use a suitable light meter to adjust the second harmonic generator (SHG) in the laser head (Figure 5) for maximum energy out.

2. If the energy is still low or if the beam is not uniform and circular, remove the laser head covers and proceed with laser cavity component alignment. NOTE: Carefully realign laser cavity components by using the horizontal and vertical 
controls on the 18 or $25 \mathrm{~cm}$, reflective-coated $(\mathrm{R} / \mathrm{C}), 100 \%$ reflecting rear mirror. During this procedure use the single shot feature on the LU660. DO NOT move any other components at this stage.

3 . If the beam cannot be rounded, a more complex realignment is necessary. For this, consult a service technician. On the other hand, the Q-switch alignment may have to be improved. The need for this is manifested as an instability in the energy output or a "snapping" sound in the system. The problem is prelasing in the oscillator caused by radiation leakage through the Q-switch before Q-switching or optical feedback from outside the oscillator. To solve the problem, slightly adjust the quarter-wave plate between the polarizer and the Pockels cell or adjust the Pockels cell itself. This adjustment should be performed with the Q-switch disabled (switch to "External" on the SB660 or, if operating on external trigger, remove the trigger source).

4. If the beam is fairly round but the energy is down after SHG adjustment, be sure that the output quarter wave plate (between the laser rod and the flat mirror) has been optimized. This optic should be adjusted VERY carefully because misadjustment can lead to trapping of energy within the cavity, resulting in optical damage.

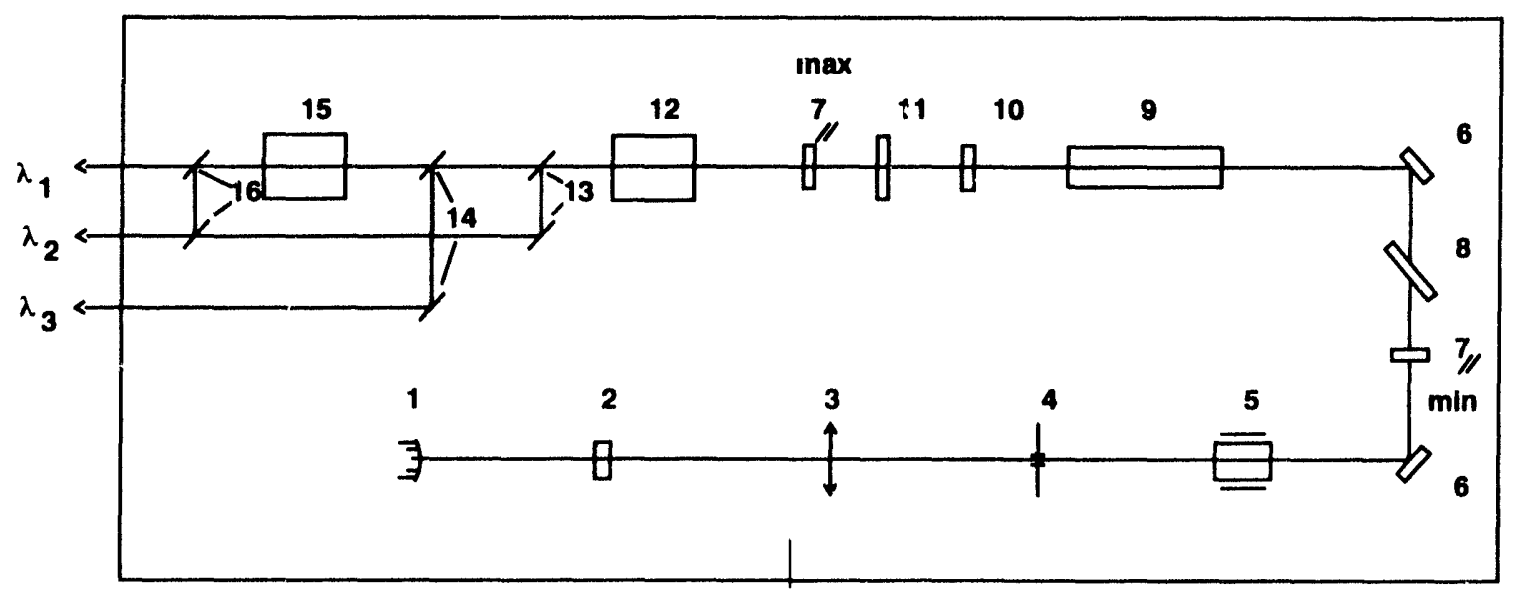

\section{LEGEND}

1. Rear mirror, $-25 \mathrm{~cm}$ for $10,1 \mathrm{z}$ $(-17.9 \mathrm{~cm}$ for $50 \mathrm{~Hz})$

2. Intracavity shutter

3. Con. lens, $+50 \mathrm{~cm}, 101-0021$

4. Pinhole

5. Pockels Cell, 202-0001

6. Turning Mirror, $45^{\circ} \mathrm{Rmax}$

7. N/4 plate, 108-0001

8. Dielectric polarizer, 199-0001

9. 611-06 head, 503-1000 $6 \times 115$ rod, 201-0056 FL611 flashlamp, 203-0019
10. N/4 plate for $20,30 \& 50 \mathrm{~Hz}$

11. Window, 1FAR, 110-0003

12. SHG, Second Harmonic Generator

13. Dichroics for $532 \mathrm{~nm}, 105-0022$

14. Dichrolcs for 1064nm, 105-0002

15. THG, Third Harmonic Generator (or Fourth)

16. Dichrolcs for $355(105-0023)$ or $266 \mathrm{nr}$, 105-0025

$\lambda_{1} .355 \mathrm{~nm}$ (not used)

$\lambda_{2} .532 \mathrm{~nm}$

$\lambda_{3} .1060 \mathrm{~nm}$

Figure 5. YG660A laser layout. 


\section{Hologram Exposure}

As indicated in the Introduction, the dual-plate, double-exposure method of hologram construction is discussed in this manual. Note that the reference becm is not aligned perpendicular to the plane of the hologram. This arrangement is called an off-axis hologram, which allows for greater separation of the diffracted hologram image and the transmitted beam during reconstruction. More detail on the off-axis technique is given later in this guide. This technique requires the YG660 Nd:YAG laser to operate in the manual (single shot) mode. The laser pulse can be iniated locally or by wiring the LU660 logic unit to fire remotely from a computer control center.

The first laser pulse should expose a plate to a uniform density test field in the tunnel before the tunnel operator opens the start valve. On the silver halide emulsion photo plate, only one face has the emulsion. For the uniform field exposure, the emulsified face is oriented toward the test field. After the start valve has been opened and the tunnel operator has achieved proper flow conditions in the tunnel, expose the second plate. This exposure will capture the nonuniform density field wave front. Now the emulsified face must be oriented opposite the test field. Perform both exposures with the laboratory lights off. It is recommended that the plates be processed as soon as possible after exposure.

\section{Hologram Plate Processing}

Hologram plate processing involves two procedures, each with a number of steps. One is the preparation of photoprocessing chemicals. The other is the processing of the photographic plates.

\section{Preparation of Photoprocessing Chemicals}

As listed in Section 3, Equipment, four mixtures of chemicals are necessary for processing $8 \mathrm{E} 56$ photographic plates. All are considered to be hazardous, and SOP No. 10204 9101, Safe Operating Procedure for Organization 1554 Film Processing Laboratory, must be read and signed before proceeding. The four mixtures are as follows: Kodak D-19 developer, Kodak indicator bath, Kodak parts A and B rapid fixers, and Kodak Photo-Flo '2u0 solution.

D-19 Developer. A 595-g packet of dry chemical (D-19) makes 3.8 L of solution.

1. Start with $3.8 \mathrm{~L}$ of water at $\sim 52^{\circ} \mathrm{C}$.

2. While stirring, slowly add the dry chemicals.

3. Stir until chemicals are dissolved and the solution is completely mixed.

4. Allow the mixture to cool to room temperature before use.

5. Store in an opaque container.

Indicator Bath. For the stop bath solution:

1. Stir $16 \mathrm{~mL}$ of Kodak stop bath indicator into $1000 \mathrm{~mL}$ of water at 18.6 to $21^{\circ} \mathrm{C}$.

2. This solution is at room temperature and therefore can be used immediately.

Rapid Fixers. The fixer solution is also mixed at room temperature $\left(18.6\right.$ to $\left.21^{\circ} \mathrm{C}\right)$ and can be stored or used as necessary. For preparation of $3.8 \mathrm{~L}$ of Kodak rapid fixer $\mathrm{A}$ and $B$ : 
1. Start with $1.9 \mathrm{~L}$ of water.

2. Add $946 \mathrm{~mL}$ of solution A.

3. While stirring rapidly, add $104 \mathrm{~mL}$ of solution $\mathrm{B}$.

4. Continue stirring, and bring the solution to $3.8 \mathrm{~L}$ with the addition of water.

5. Stir until solution is uniform.

Photo-Flo 200. To produce a 1:200 parts ratio of Photo-Flo 200 solution:

1. Mix $10 \mathrm{ml}$ Kodak Photo-Flo 200 solution with $2000 \mathrm{~mL}$ water.

2. Stir.

This completes the necessary photochemical preparations, and with four stainless steel trays, photoprocessing can begin.

\section{Processing of Photographic Plates}

Practice this procedure in darkness to gain familiarity before attempting to actually expose plates. The time used for each step may vary depending on wind tunnel flow field characteristics or hologram exposure during construction. Perform the following procedure in red light if $8 \mathrm{E} 56$ is used since this wavelength is beyond the sensitivity of the emulsion. If 8E56 is not used, complete darkness is required.

1. First immerse the plate(s) in a tray with at least 1 in. of D-19 developer solution.

2. Carefully move plate(s) around in solution for 3 to $4 \mathrm{~min}$.

3. Remove plate(s) from D-19 solution.

4. Place plate(s) in a tray of stop bath solution for $30 \mathrm{~s}$.

5. Remove plate(s) and place in a tray of $1 \mathrm{in}$. deep fixer solution for $4 \mathrm{~min}$.

6. Remove plate(s) from fixer solution.

7. Place plate(s) in a half filled tray of Kodak Photo-Flo 200 solution for 15 to $25 \mathrm{~min}$.

8. Remove and dry the plate(s) by wiping with a clean, dry cloth.

As a reminder, all waste chemicals from this procedure are hazardous and must be disposed of in accordance with SOP No. 102049101.

\section{Hologram Image Reconstruction}

Hologram image reconstruction is the final stage of optical manipulation in the holographic interferometry procedure. Figure 6 is a schematic diagram of the hologram reconstruction optics. The laser source used is a Model 164 argon ion laser, which is a continuous wave Class IV laser. Observe all proper safety considerations for Class IV laser operation while performing hologram image reconstruction.

Caution: Protect your eyes properly and adhere to SOP 118008904 before continuing with this procedure.

The optical system used for hologram reconstruction is basically the same as the reference beam path used in hologram construction (Figures 2 and 3 ). In fact, the angle of incidence of the reconstruction beam on the hologram plates is the same as that used between the construction hologram and the reference beam. This off-axis arrangement produces an image on the opposite side of the hologram plates at a complementary angle to the normal of the plates, allowing separation between the image and transmitted beam. At this location, erect a frosted glass plate to diffusely disperse the image so that it can be captured with a video camera and transferred to the video digitizer system for storage. 


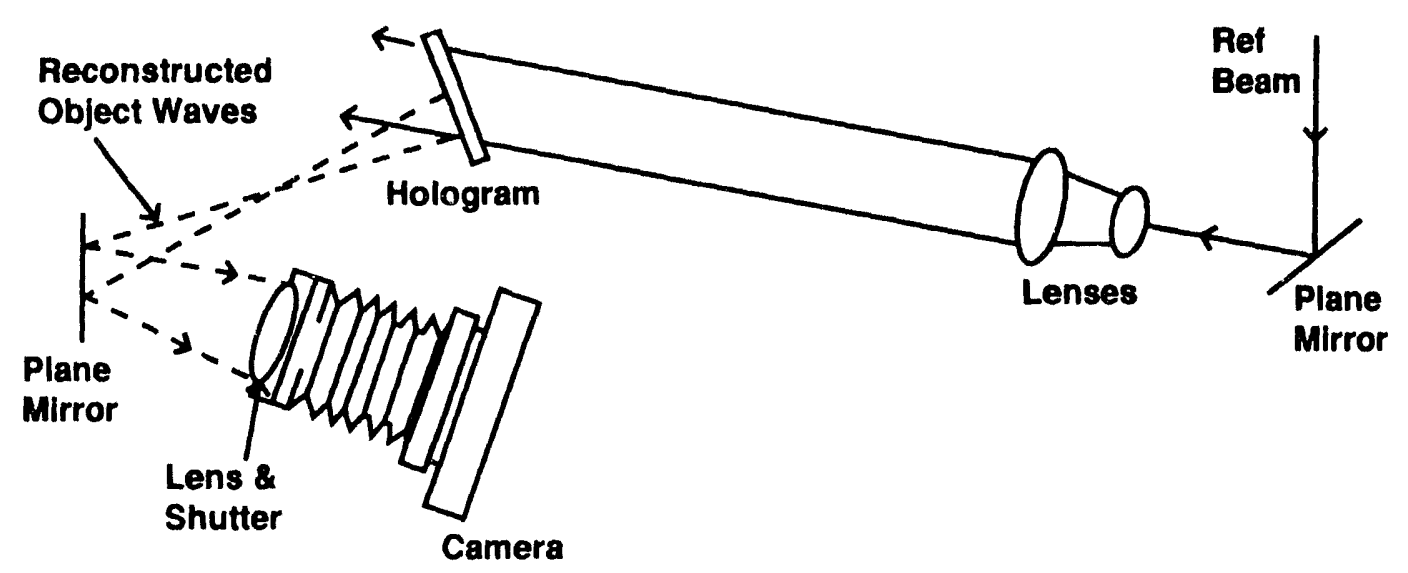

Figure 6. Hologram playback system.

Arrange the developed uniform and nonuniform density field plates in the plate holder with the same relative position as in the construction procedure: the emulsified surfaces outward and the nonemulsified surfaces inward. Adjust the plate holder used for reconstruction with piezoelectric micropositioners to accurately control the angle and relative position between the plates.

\section{Image Storage and Analysis}

After capturing an image with a video digitizer and storing it on the image processing system hard disk drive, use the IrnagePro software to enhance image contrast or filter the image to produce the optimum conditions for analysis. Figure 7 is an example of a holographic image.

\section{Data Reduction of the Holographic Interferogram}

Final data reduction of the holographic interferogram can take one of three forms:

- Visual araalysis

- Interferometric analysis

- Computerized tomographic (CT) analysis.

Make a visual analysis if you have some knowledge of the density field, for example, if the model geometry is simple or the flow fields are symmetric. If a more exact and detailed analysis is needed, use interferometric techniques that involve measurement of changes in the physical properties of transparent objects (Figure 8). Such properties include mass density of fluids, electron density of plasmas, temperature of fluids, chemical species concentrations in reacting gases, and state of stress in solids. Each of these properties can be related to the refractive index, which is measured by interferometry. A very interesting and complete analysis can be performed through computerized tomography, which is a mathematical, three dimensional reconstruction of the wind tunnel flow field. The greatest amount of information can be acquired by using this method. 


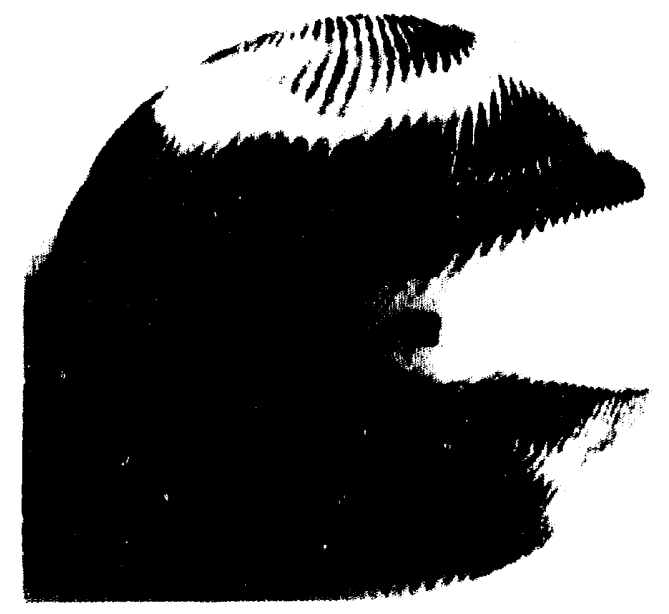

Figure 7. Hologram image and fringe pattern.

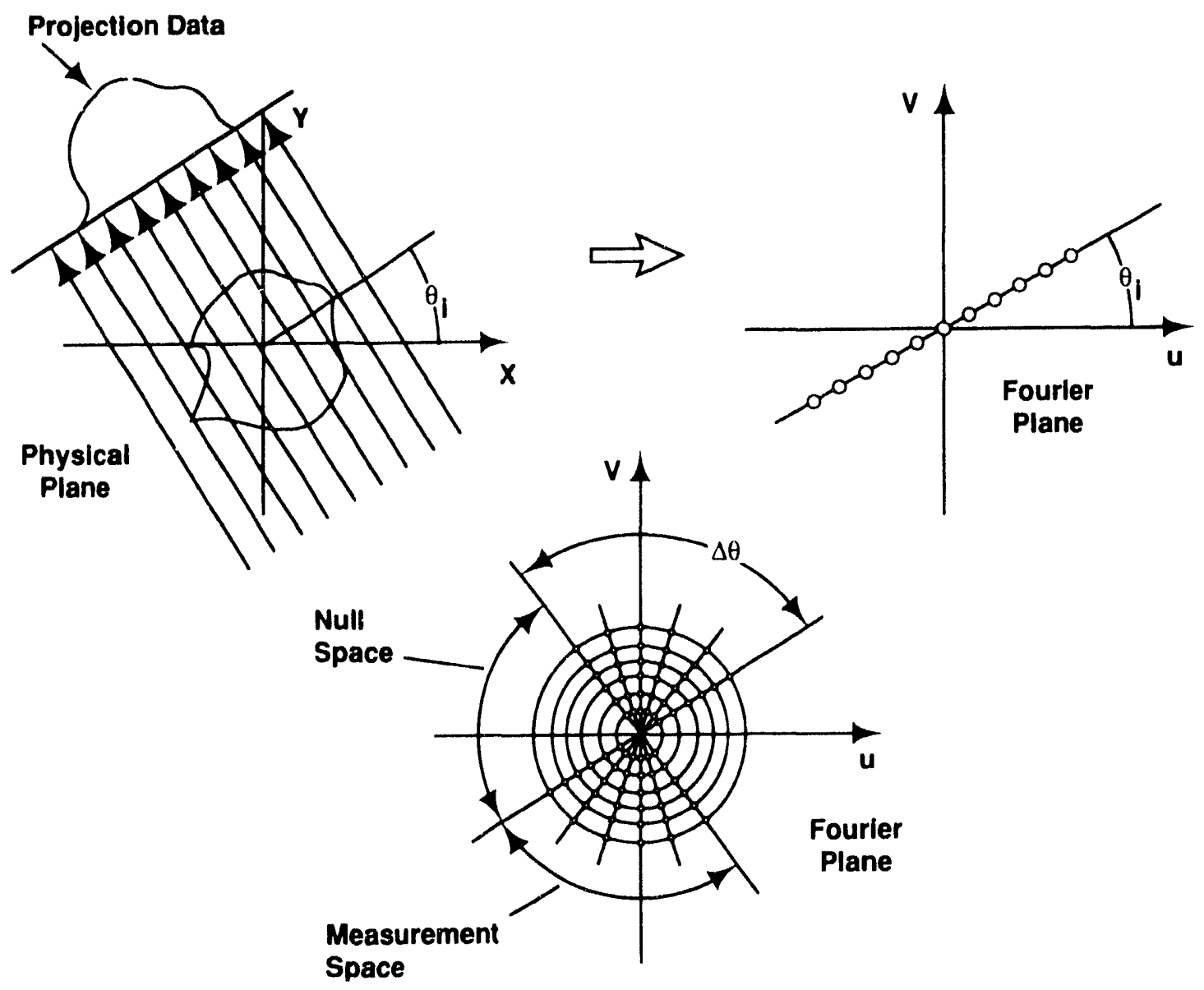

Figure 8. Relationship of physical and Fourier planes. 


\section{Problem Areas in Holographic Interferometry}

Holographic interferometry allows workers in the field of aerodynamics a much more exact and detailed analysis of flow field characteristics than is otherwise possible. In addition, it will broaden the scope of analysis by allowing reconstruction of complex, asymmetric density fields in three dimensions. Yet several areas can pose operational problems.

\section{Transverse Multimodes}

One problem area is the transverse multimodes in Nd:YAG lasers. This phenomenon manifests itself in the laser cavity. The simplest mode in a cavity is described by a ray that travels back and forth along the axis. This is the 00 mode. Because of diffraction, the actual intensity distribution is that shown in Figure 9.

The output of a laser oscillating in this mode is a spherical wave with a Gaussian intensity distribution. The beamwidth is usually expressed as the radius $w$, at which the beam intensity falls to $1 / \mathrm{e}^{2}$ of its maximum value. Higher order modes correspond to closed paths with yet higher numbers of reflections required to complete a round trip. Transverse-mode patterns are labeled according to the number of minima (darker areas) that are encountered when the beam is scanned horizontally (first number) and then vertically (second number). This nonhomogeneity of beam intensity will show on the developed hologram and make fringe interpretation and measurement very difficult.

These mode oscillations cannot be controlled in the laser, but with image fast Fourier transforms (FFTs) and digital filtering, the affected hologram can be used and fringe interpretation accomplished (Caulfied, 1979).

\section{Laser Rod and Component Temperature}

As mentioned earlier, beam energy and geometry are important for hologram construction. These parameters are greatly affected by laser rod and component temperature, which change throughout laser operation. As a result, the laser will need to be realigned and adjusted while a wind tunnel test run is conducted. This problem can be minimized but will always have $t$, he dealt with.

\section{Relative Lightness and Darkness}

Finally, since relative lightness and darkness in the reconstructed image is the primary means of determining the fringe configuration and pattern, do not use a camera with automatic features that will vary the output signal to the digitizer depending on the range of intensities in the reconstructed image. Some features to be avoided are automatic iris adjustment, automatic focus adjustment, and automatic voltage range adjustment, based on the relative contrast from the image. Therefore, a simple video camera is recommended. 

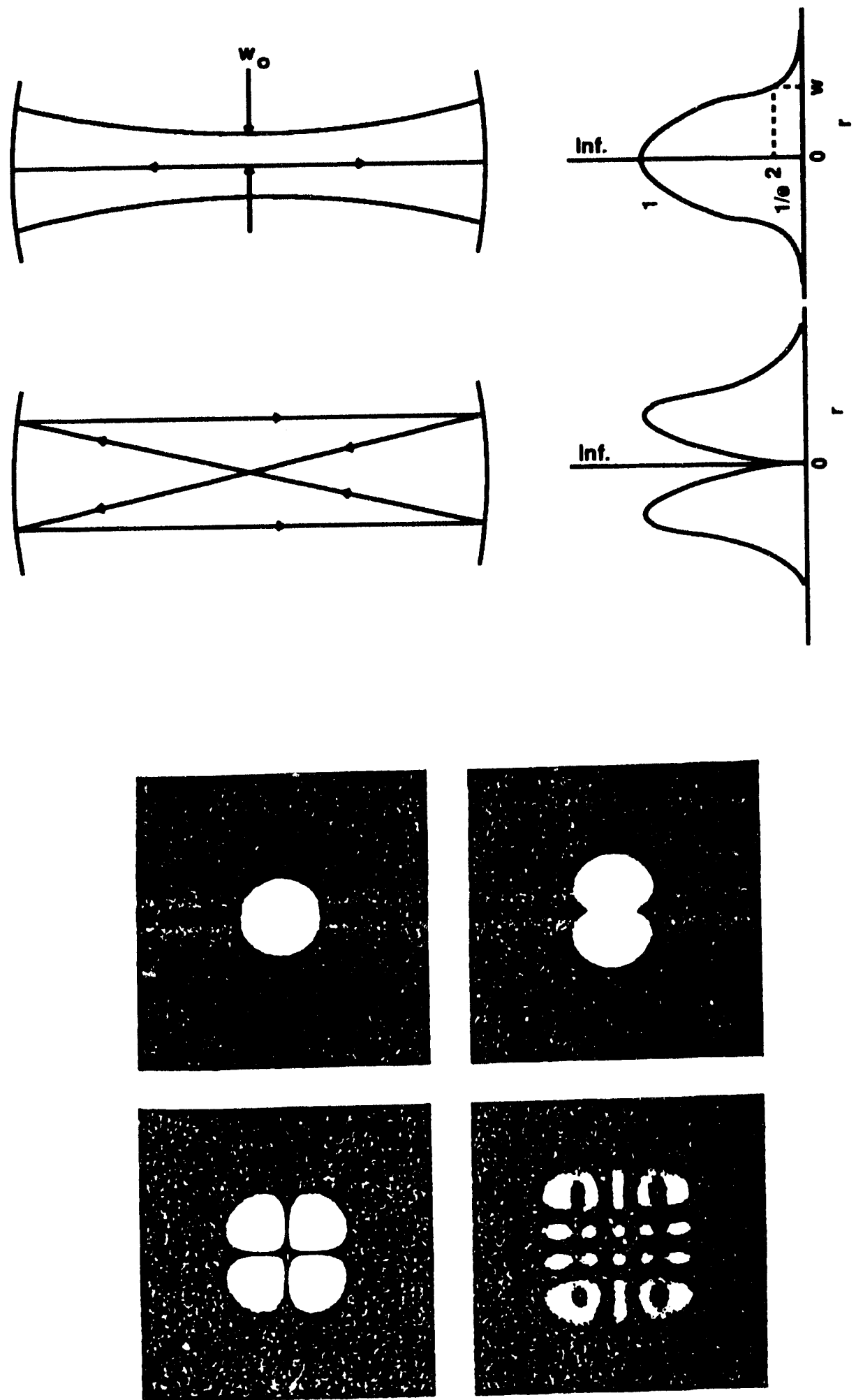

Figure 9. Transverse modes in a laser cavity $(00.01$ shown here schematically; from Model YG660A Laser Technical Manual, Quantel International, 1988). $\mathrm{W}_{\mathrm{o}}=$ Beam waist: Location in the beam where beam energy is $1 / \mathrm{e}^{2}$ of the energy at the center; also marks the spot of common focus of two end mirrors. 


\section{7. "Conclusion}

With the increased availability of faster computers with higher memory capacity, image processing for use with holographic interferograms is a practical and necessary addition to the aerodynamic engineering field. Image and fringe interpretation can be greatly enhanced by determining what specific digital filter to use on each image. Individual image background fields may change from exposure to exposure for reasons that are uncontrollable during hologram construction. Restarting the complete test can be expensive, both in time used and money spent. With a "hard copy" of flow field characteristics anc digital enhancement, less emphasis can be placed on perfect hologram construction.

Finally, with computerized tomography, more accurate and detailed flow field data can be extracted from a given modei, providing a way to calibrate computer simulations of model flow fields. Thus, a more reliable database will be available for future engineering use. 


\section{Bibliography}

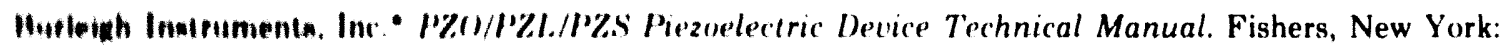
Burleigh Inetruments. IGA6

1.ulliwid. II I, ad Handtumk of Iptical Holography. New York: Academic Press, 1979.

Thete. Niteve "Safe Operatine Procedure Finclosed Area Around Hypersonic Wind Tunnel in Bldg 865,"

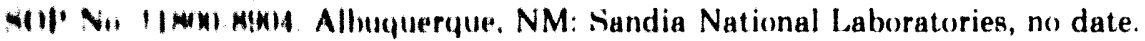

Vatteter the Mathemates of cumputerized Tomography. New York: John Wiley \& Sons, 1986.

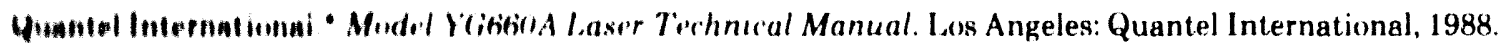

Hin hwoll Amerutem. Inr Laxer Sofoly. Training Manual. Cincinnati: Rockwell Associates, 1983.

Nmith II M. ml Holinraphic Recording Materuals. New York: Springer-Verlag, 1977.

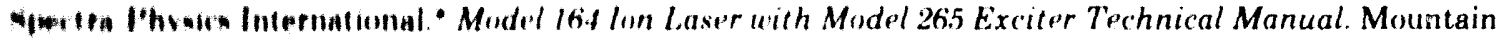
Vion I'A simetra l'hyesics. 197is.

- Ieforence to a commercial product does not imply endorsement by Sandia National Laboratories or the lenartmem of Finergy of the lack of a sultable substitute. 


\section{DISTRIBUTION:}

$\begin{array}{rll}1 & 1040 & \text { R. T. Johnson } \\ 1 & 1041 & \text { L. J. Azevedo } \\ 10 & 1042 & \text { D. Griggs } \\ 1 & 1042 & \text { R. B. Pettit } \\ 1 & 1043 & \text { B. E. Barnaby } \\ 1 & 1512 & \text { T. J. O'Hern } \\ 1 & 1553 & \text { V. A. Amatucci } \\ 1 & 1554 & \text { D. P. Aeschliman } \\ 1 & 1554 & \text { W. H. Rutledge } \\ 1 & 8523-2 & \text { Central Technical Files } \\ 5 & 7141 & \text { Technical Library } \\ 1 & 7151 & \text { Technical Publications } \\ 10 & 7613-2 & \text { Document Processing } \\ & & \text { For DOE/OSTI }\end{array}$



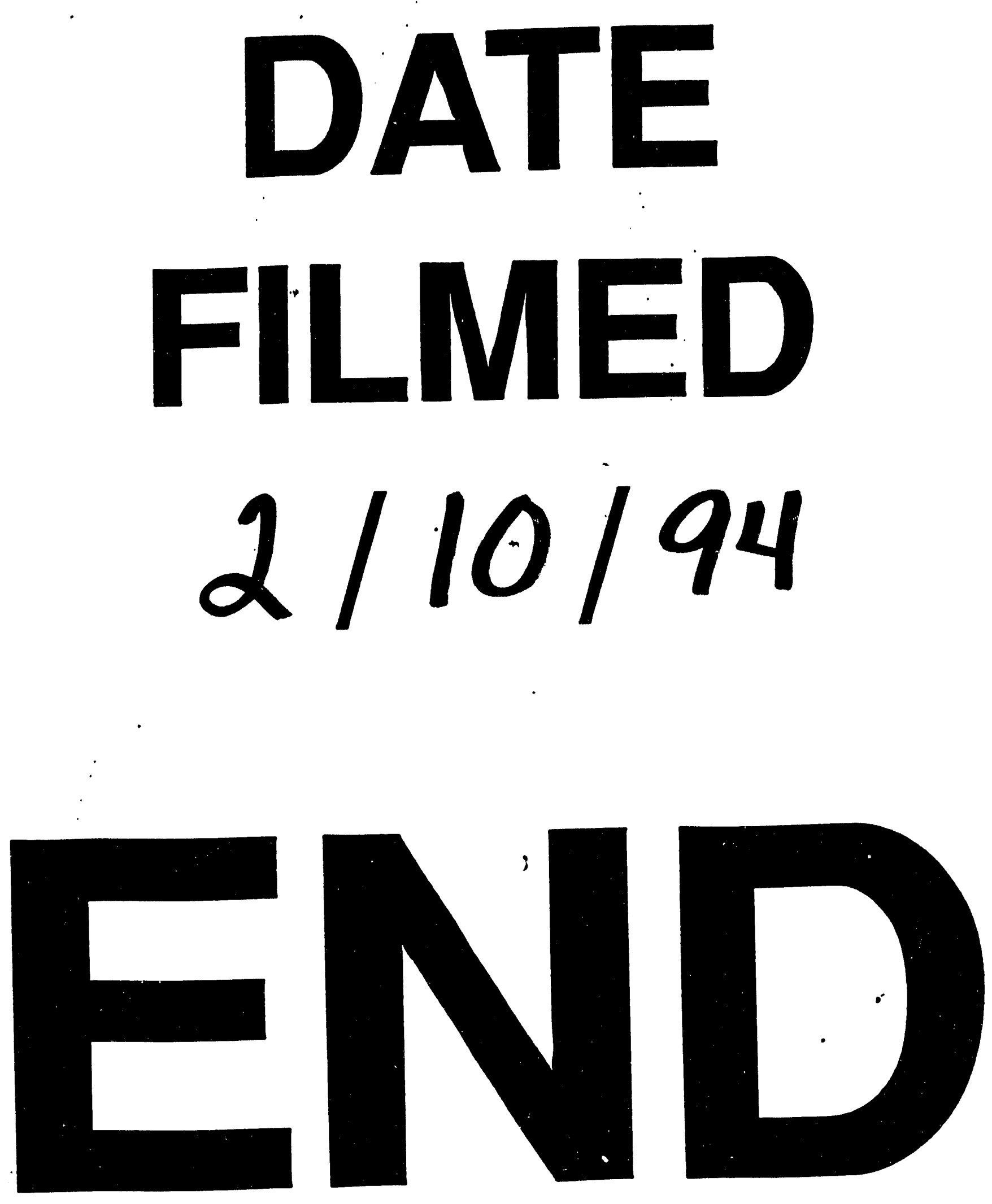
